\title{
Dynamic model identification of IPMC actuator using fuzzy NARX model optimized by MPSO
}

- Ho Pham Huy Anh

FEEE, University of Technology, VNU-HCM

- Nguyen Thanh Nam

DCSELAB, University of Technology, VNU-HCM

(Manuscript Received on December 11 ${ }^{\text {th }}, 2013$; Manuscript Revised September 12 $2^{\text {th }}, 2014$ )

\begin{abstract}
:
In this paper, a novel inverse dynamic fuzzy NARX model is used for modeling and identifying the IPMC-based actuator's inverse dynamic model. The contact force variation and highly nonlinear cross effect of the IPMC-based actuator are thoroughly modeled based on the inverse fuzzy NARX model-based identification process using experiment input-output training data. This

paper proposes the novel use of a modified particle swarm optimization (MPSO) to generate the inverse fuzzy NARX (IFN) model for a highly nonlinear IPMC actuator system. The results show that the novel inverse dynamic fuzzy NARX model trained by MPSO algorithm yields outstanding performance and perfect accuracy.
\end{abstract}

Keywords: IPMC-based actuator, modified particle swarm optimization (MPSO), fuzzy NARX model, inverse dynamic identification

\section{INTRODUCTION}

The nonlinear IPMC-based actuator is belonged to highly nonlinear systems where perfect knowledge of their parameters is unattainable by conventional modeling techniques because of the time-varying inertia, external force variation and other nonlinear uncertainties. To guarantee a good position tracking performance, lots of researches have been carried on. During the last decade, Sadeghipour et al., Shahinpoor et al., Oguru et al., and Tadokoro et al. investigated the bending characteristics of Ionic Polymer Metal Composite (IPMC) [1-4]. Bar-Cohen et al. characterized the electromechanical properties of IPMC [5]. An empirical control model by Kanno et al. was developed and optimized with curve-fit routines based on open-loop step responses with three stages, i.e., electrical, stress generation, and mechanical stages [6-8]. Feedback compensators were designed using a similar model in a cantilever configuration to study its open-loop and closed-loop behaviors [9-10]. 
Damping of the ionic polymer actuator in air is much lower than that in water. Feedback control is necessary to decrease the response time of an ionic-polymer actuator to a step change in the applied electric field and to reduce overshoot. The position control of the IPMC was investigated by using a linear quadratic regulator (LQR) [12], a PID controller with impedance control [11], and a lead-lag compensator [9-10]. Lots of advanced control algorithms have been developed for IPMC actuator in order to apply them in variety of the industrial and marine applications [13-19].

Up to now, the robust-adaptive control approaches combining conventional methods with new learning techniques are realized. During the last decade several neural network models and learning schemes have been applied to offline and online learning of actuator dynamics. Ahn and Anh in [20] have successfully optimized a NARX fuzzy model of the highly nonlinear actuator using genetic algorithm. These authors in [21] have identified the nonlinear actuator based on recurrent neural networks. The drawback of all these results is related to consider the actuator as an independent decoupling system and the external force variation like negligible effect. Consequently, all intrinsic cross-effect features of the IPMC-based actuator has not represented in its intelligent model. Recently, D.N.C. Nam et al. has modeled the IPMC actuator using fuzzy model optimized by traditional PSO [22-23]. The drawback of this research lied in the resulting fuzzy model optimized by the traditional PSO susceptible to premature convergence and then easy to be fallen in local optimal trap.

In order to overcome this disadvantage, this paper proposes the novel use of a modified particle swarm optimization (MPSO) to generate the inverse fuzzy NARX (IFN) model for a highly nonlinear IPMC actuator system. The MPSO is used to process the experimental input-output data that is measured from the IPMC system to optimize all nonlinear and dynamic features of this system. Thus, the MPSO algorithm optimally generates the appropriate fuzzy if-then rules to perfectly characterize the dynamic features of the IPMC actuator system. These good results are due to proposed IFN model combines the extraordinary approximating capability of the fuzzy system with the powerful predictive and adaptive potentiality of the nonlinear NARX structure that is implied in the proposed IFN model. Consequently, the proposed MPSO-based IPMC inverse fuzzy NARX model identification approach has successfully modeled the nonlinear dynamic IPMC system with better performance then other identification methods.

This paper makes the following contributions: first, the novel proposed MPSO-based IPMC inverse fuzzy NARX model for modeling and identifying dynamic features of the highly nonlinear IPMC system has been realized; second, the modified particle swarm optimization (MPSO) has been applied for optimizing the IPMC IFN model's parameters; finally, the excellent results of proposed IPMC inverse fuzzy NARX model optimized by MPSO were obtained.

The rest of the paper is organized as follows. 
Section 2 introduces the novel proposed inverse fuzzy NARX model. Section 3 presents the experimental set-up configuration for the proposed IPMC IFN model identification. Section 4 describes concisely the modified particle swarm optimization (MPSO) used to identify the IPMC IFN model. Section 5 is dedicated to the techniques of MPSO-based IFN model identification. The results from the proposed IPMC IFN model identification are presented in Section 6. Section 7 contains the concluding remarks.

\section{PROPOSED INVERSE FUZZY NARX MODEL OF NONLINEAR IPMC SYSTEM}

\subsection{Proposed inverse fuzzy NARX model of the IPMC actuator system}

The proposed IFN model of the highly nonlinear IPMC system presented in this paper is improved by combining the approximating capability of the fuzzy system with the powerful predictive and adaptive potentiality of the nonlinear NARX structure. The resulting model establishes a nonlinear relationship between the past inputs and outputs and the predicted output, while the system prediction output is a combination of the system output produced by the real inputs and the historical behaviors of the system. This can be expressed as:

$$
\hat{y}(k)=f\left(y(k-1), \ldots, y\left(k-n_{a}\right), u\left(k-n_{d}\right), \ldots, u\left(k-n_{b}-n_{d}\right)\right)
$$

Here, na and $\mathrm{nb}$ are the maximum lag considered for the output and input terms, respectively, nd is the discrete dead time, and $\mathrm{f}$ represents the mapping of the fuzzy model.

The structure of the proposed IPMC IFN model interpolates between the local linear, timeinvariant (LTI) ARX models as:

Rule $\mathrm{j}$ : if $\mathrm{zl}(\mathrm{k})$ is $\mathrm{A} 1, \mathrm{j}$ and $\ldots$ and $\mathrm{zn}(\mathrm{k})$ is An,j then

$\hat{y}_{j}(k)=\sum_{i=1}^{n_{a}} a_{i}^{j} y_{j}(k-i)+\sum_{i=1}^{n_{b}} b_{i}^{j} u\left(k-i-n_{d}\right)+c^{j}$

where $\mathrm{zi}(\mathrm{k}), \mathrm{i}=1 \ldots \mathrm{n}$ is the element of the $\mathrm{Z}(\mathrm{k})$ "scheduling vector" which is usually a subset of the $\mathrm{X}(\mathrm{k})$ regressor that contains the variables relevant to the nonlinear behaviors of the system. In this paper, $\mathrm{X}(\mathrm{k})$ regressor contains all of the inputs of the inverse fuzzy NARX model

$Z(k) \in X(k)=\left\{y(k-1), \ldots, y\left(k-n_{a}\right), u\left(k-n_{d}\right), \ldots, u\left(k-n_{b}-n_{d}\right)\right\}$

The $f j(q(k))$ consequent function contains all the regressors $\mathrm{q}(\mathrm{k})=[\mathrm{X}(\mathrm{k}) 1]$,

$f_{j}(q(k))=\sum_{i=1}^{n_{a}} a_{i}^{j} y(k-i)+\sum_{i=1}^{n_{b}} b_{i}^{j} u\left(k-i-n_{d}\right)+c^{j}$

In the simplest case, the NARX type zero-order fuzzy model (singleton or Sugeno fuzzy model which isn't applied in this paper) is formulated by the simple rule consequents:

Rule $\mathrm{j}$ : if $\mathrm{zl}(\mathrm{k})$ is $\mathrm{A} 1, \mathrm{j}$ and... and $\mathrm{zn}(\mathrm{k})$ is An,j then

$$
\hat{y}(k)=c^{j}
$$

with $\mathrm{zi}(\mathrm{k}), \mathrm{i}=1 \ldots \mathrm{n}$ is the element of the $\mathrm{Z}(\mathrm{k})$ regressor containing all of the inputs of the IPMC IFN model:

$Z(k)=X(k)=\left\{y(k-1), \ldots, y\left(k-n_{a}\right), u\left(k-n_{d}\right), \ldots, u\left(k-n_{b}-n_{d}\right)\right\}$

Thus the difference between the fuzzy NARX model and the classic TS Fuzzy model method is that the output from the TS fuzzy model is linear and constant, and the output from the NARX 
fuzzy model is the NARX function. However, both of these methods have the same fuzzy inference structure (FIS).

\subsection{MPSO-based IPMC IFN Model Identification}

The problem of modeling the nonlinear and dynamic system always attracts the attention of researcher. Some research has been published using a fuzzy model based on expert knowledge [24-30]. Unfortunately the resulting fuzzy model was often too complex to be applied in practice and thus only simulation was carried out. Figure $1 \mathrm{a}$ and $1 \mathrm{~b}$ initially presents the block scheme for the modeling and identification of a MPSO-based inverse fuzzy NARX11 and inverse fuzzy NARX22 models using experimental input-output training data. MPSO stands for Modified Particle Swarm Optimization and will be described later in the section 4.1 .

This proposed approach can help to simplify the modeling procedure for nonlinear systems. Particle swarm optimization (PSO) is applied to optimize the FIS structure and other parameters of proposed fuzzy model. However the poor experimental result proves that the PSO-based TS fuzzy model is incapable of modeling all nonlinear, dynamic features of the dynamic system. Recently the fuzzy/neural NARX model has been successfully applied to identify nonlinear, dynamic system [20],[27].

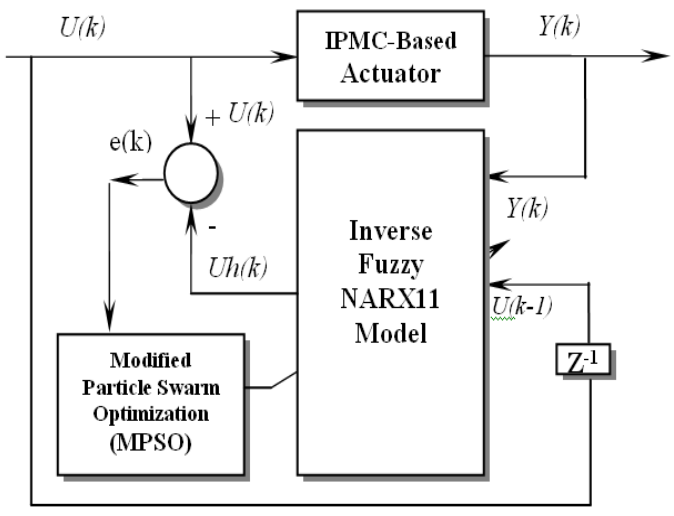

Fig.1. Block diagram of the MPSO-based IPMC inverse fuzzy NARX11 model identification

The block diagram presented in Fig. 1 and 2 illustrate the MPSO-based IPMC IFN model identification. The error $\mathrm{e}(\mathrm{k})=\mathrm{U}(\mathrm{k})-\mathrm{Uh}(\mathrm{k})$ is used by the MPSO algorithm to calculate the Fitness value (see Equation (7)) in order to identify and optimize parameters of the proposed IPMC IFN model.

$$
F_{j}=10^{4} .\left(\frac{1}{M} \sum_{k=1}^{M}\left(y(k)-\hat{y}_{j}(k)\right)^{2}\right)^{-1}
$$

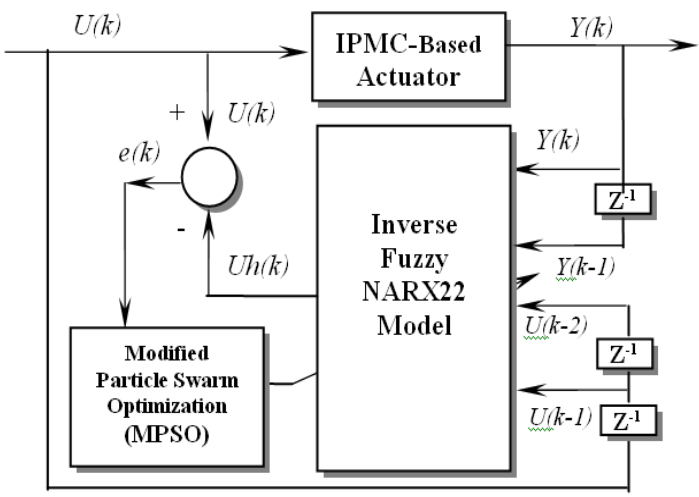

Fig.2. Block diagram of the MPSO-based IPMC inverse fuzzy NARX22 model identification

\section{EXPERIMENT CONFIGURATION OF} THE IPMC IFN MODEL IDENTIFICATION

A general configuration and the schematic diagram of the IPMC-based actuator and the 
photograph of the experimental apparatus are shown in Fig.3.

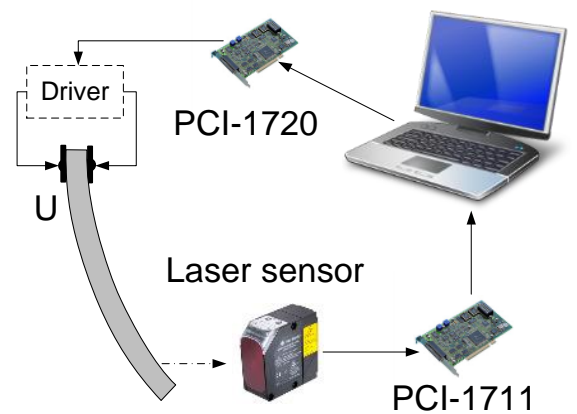

Fig.3. Block diagram for working principle of IPMC actuator inverse fuzzy NARX model identification

The hardware includes an IBM compatible PC (Pentium 1.7 GHz) which sends the voltage signals $\mathrm{u}(\mathrm{t})$ to control the proportional valve (FESTO, MPYE-5-1/8HF-710B), through a D/A board (ADVANTECH, PCI 1720 card) which changes digital signals from PC to analog voltage $\mathrm{u}(\mathrm{t})$ respectively. The rotating torque is generated by the pneumatic pressure difference supplied from air-compressor between the antagonistic artificial muscles. Consequently, the both of joints of the IPMC-based intelligent valve will be rotated to follow the desired joint angle references (YREF1(k) and YREF2(k)) respectively.

\section{PSO ALGORITHM FOR NARX FUZZY MODEL IDENTIFICATION}

PSO is a population-based optimization method first proposed by Eberhart and colleagues [32] Some of the attractive features of PSO include the ease of implementation and the fact that no gradient information is required. It can be used to solve a wide array of different optimization problems. Like evolutionary algorithms, PSO technique conducts search using a population of particles, corresponding to individuals. Each particle represents a candidate solution to the problem at hand. In a PSO system, particles change their positions by flying around in a multidimensional search space until computational limitations are exceeded. Concept of modification of a searching point by PSO is shown in Fig. 4.

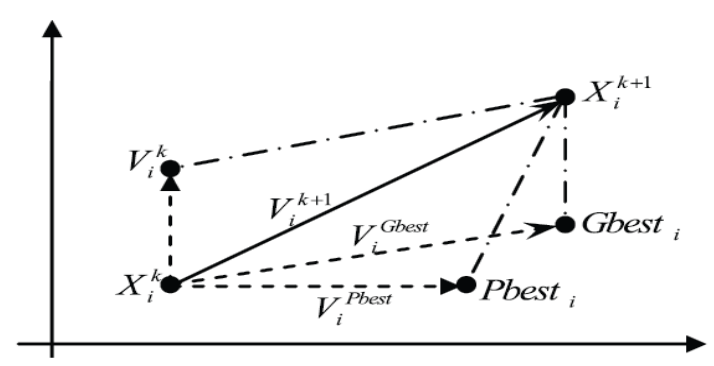

Fig. 4. Searching Concept of PSO

With:

$\mathrm{Xk}$ : current position, $\mathrm{Xk}+1$ : modified position, Vk: current velocity, $\mathrm{Vk}+1$ : modified velocity, VPbest: velocity based on Pbest, VGbest: velocity based on Gbest.

The PSO technique is an evolutionary computation technique, but it differs from other well-known evolutionary computation algorithms such as the genetic algorithms. Although a population is used for searching the search space, there are no operators inspired by the human DNA procedures applied on the population. Instead, in PSO, the population dynamics simulates a 'bird flock's' behavior, where social sharing of information takes place and individuals can profit from the discoveries and previous experience of all the other companions during the search for food. Thus, each companion, called particle, in the population, which is called swarm, is assumed to 'fly' over the search space in order to find 
promising regions of the landscape. For example, in the minimization case, such regions possess lower function values than other, visited previously. In this context, each particle is treated as a point in a d-dimensional space, which adjusts its own 'flying' according to its flying experience as well as the flying experience of other particles (companions)

In PSO, a particle is defined as a moving point in hyperspace. For each particle, at the current time step, a record is kept of the position, velocity, and the best position found in the search space so far. The assumption is a basic concept of PSO [32]. In the PSO algorithm, instead of using evolutionary operators such as mutation and crossover, to manipulate algorithms, for a dvariable optimization problem, a flock of particles are put into the d-dimensional search space with randomly chosen velocities and positions knowing their best values so far (Pbest) and the position in the d-dimensional space. The velocity of each particle, adjusted according to its own flying experience and the other particle's flying experience. For example, the i-th particle is represented as $\mathrm{xi}=(\mathrm{xi}, 1, \mathrm{xi}, 2, \ldots, \mathrm{xi}, \mathrm{d})$ in the $\mathrm{d}$ dimensional space. The best previous position of the i-th particle is recorded and represented as:

Pbesti $=($ Pbesti,1, Pbesti,2 ,..., Pbesti,d).

The index of best particle among all of the particles in the group in the d-dimensional space is gbestd. The velocity for particle $\mathrm{i}$ is represented as $\mathrm{vi}=(\mathrm{vi}, 1, \mathrm{vi}, 2, \ldots, \mathrm{vi}, \mathrm{d})$. The modified velocity and position of each particle can be calculated using the current velocity and the distance from
Pbesti,d to gbestd as shown in the following formulas [37]:

$v_{i, m}^{(t+1)}=w v_{i, m}^{(t)}+c_{1}$ Rand (). $\left[\right.$ Pbest $\left.t_{i, m}-x_{i, m}^{(t)}\right]+c_{2}$ Rand (). $\left[\right.$ gbest $\left._{m}-x_{i, m}^{(t)}\right]$

$x_{i, m}^{(t+1)}=x_{i, m}^{(t)}+v_{i, m}^{(t+1)}, \quad i=1,2, \ldots, n ; m=1,2, \ldots, d$

where

$\mathrm{n}$ - Number of particles in the group,

$\mathrm{d}$ - Dimension of search space of PSO,

$\mathrm{t}$ - Pointer of iterations (generations),

$v_{i, m}^{(t)}$-Velocity of particle $\mathrm{i}$ at iteration $\mathrm{t}$,

w - Inertia weight factor,

c1, c2 - Acceleration constant,

rand() - Random number between 0 and 1 ,

$x_{i, d}^{(t)}$ - Current position of particle i at iteration $\mathrm{t}$,

Pbesti - Best previous position of the i-th particle,

Gbest-Best particle among all the particles in the population

The evolution procedure of PSO Algorithms is shown in Fig. 5. Producing initial populations is the first step of PSO. The population is composed of the chromosomes that are real codes. The corresponding evaluation of a population is called the "fitness function". It is the performance index of a population. The fitness value is bigger, and the performance is better. The fitness function is defined as equation (7).

After the fitness function has been calculated, the fitness value and the number of the generation determine whether or not the evolution procedure is stopped (Maximum iteration number reached?). 
In the following, calculate the Pbest of each particle and Gbest of population (the best movement of all particles). The update the velocity, position, gbest and pbest of particles give a new best position.

In recent years, the PSO has continued to be improved upon and has been applied successfully to identify and optimize different nonlinear, dynamic systems [33-34]. However the inappropriate choice of operators and parameters used in PSO process makes the PSO susceptible to premature convergence.

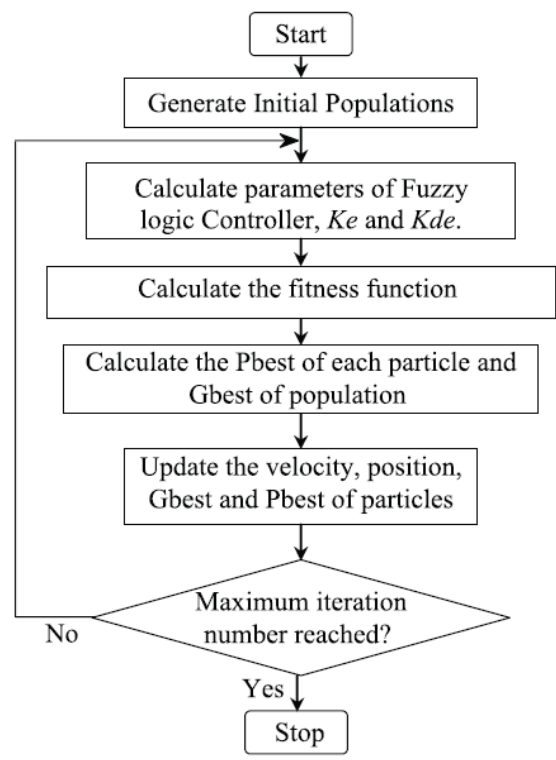

Fig. 5. Evolutionary Procedure of PSO Algorithms

The focus of this paper is to attempts to simultaneously apply two improved strategies as a means to overcome these problems.

Extinction strategy: This technique prevents the searching process from being trapped at a local optimum. Based on this concept, after Le generations, if no further increase in the fitness value has been detected; i.e., variance equal to zero, then the best $\mathrm{q} \%$ of particles survive according to their better fitness values. The others are randomly generated to fill out the population. For those surviving particles, they are allowed to mate as usual to form the next generation.

Elitist strategy: When creating a new population by crossover and mutation, it may cause to lose the best particles. The advanced elitist strategy guarantees not only the survival of the best particle in a generation but also assures that the search space is widely modified by mutating the worst particle with a higher mutation rate. Thus, this strategy ensures the continuous increase of the maximum fitness value from generation to generation. Consequently, proposed advanced elitism can rapidly increase the performance of the PSO, because it prevents loss of the best solution and asserts the higher probability in searching for the global optimum.

The proposed Modified Particle Swarm Optimization (MPSO) adopts all of the advanced strategies that were used to modify the classic PSO. The elitist strategy ensures a steady increase in the maximum fitness value. The extinction strategy prevents the searching process from becoming trapped in local optima. Consequently, the overall efficiency and the optimum solution are greatly improved when these modifications are used.

\section{MPSO-BASED INVERSE FUZZY NARX MODEL IDENTIFICATION TECHNIQUE}

\subsection{Assumptions and Constraints}

The first assumption is that symmetrical membership functions about the $y$-axis will 
provide a valid fuzzy model. A symmetrical rule-base is also assumed. Other constraints are also introduced to design the Inverse NARX Fuzzy (IMNF) Model.

* All universes of discourses are normalized to lie between -1 and 1 with scaling factors external to the IDNFM which is used to assign appropriate values to the input and output variables.

* It is assumed that the first and last membership functions have their apexes at -1 and 1 , respectively. This can be justified by the fact that changing the external scaling would have a similar effect to changing these positions.

* Only triangular membership functions are to be used.

* The number of fuzzy sets is constrained to be an odd integer that is greater than unity. In combination with the symmetry requirement, this means that the central membership function for all variables will have an apex at zero.

* The base vertices of the membership functions are coincident with the apex of the adjacent membership functions. This ensures that the value of any input variable is a member of at most two fuzzy sets, which is an intuitively sensible situation. It also ensures that when a variable's membership of any set is certain, i.e. unity, it is a member of no other sets.

Using these constraints the design of the IMNF model's input and output membership functions can be described using two parameters which include the number of membership functions and the positioning of the triangle apexes.

\subsection{Spacing parameter}

The second parameter specifies how the centers are spaced out across the universe of discourse. A value of one indicates even spacing, while a value larger than unity indicates that the membership functions are closer together in the center of the range and more spaced out at the extremes as shown in Fig.6. The position of each center is calculated by taking the position of where the center would be if the spacing were even and by raising this to the power of the spacing parameter. For example, in the case where there are five sets, with even spacing $(p=1)$ the center of one set would be at 0.5 . If $p$ is modified to two, the position of this center moves to 0.25 . If the spacing parameter is set to 0.5 , this center moves to $(0.5) 0.5=0.707$ in the normalized universe of discourse. Fig. 6 shows the triangle input membership function with spacing factor $=0.5$.

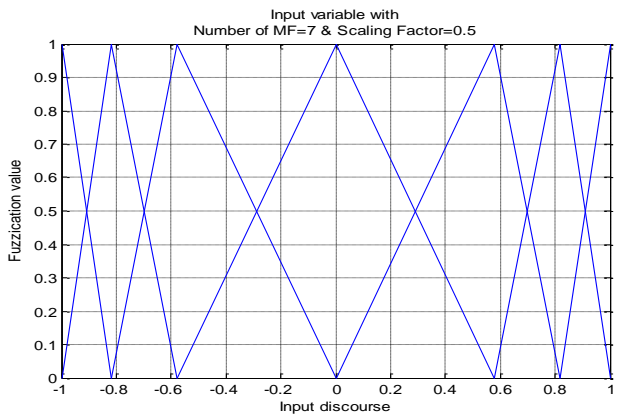

Fig.6. Triangle input membership function with spacing factor of 0.5 .

\subsection{Designing the rule base}

In addition to specifying the membership functions, the rule-base also needs to be designed. Cheong's idea was applied [34]. In specifying a rule base, both the characteristic 
spacing parameters for each variable and the characteristic angle for each output variable were used to construct the rule-base.

Certain characteristics of the rule-base are assumed when the proposed construction method is used:

$* \square \square$ Extreme outputs usually occur more often when the inputs have extreme values while the mid-range outputs are generally generated when the input values are also mid-range.

* $\square \square$ Similar combinations of input linguistic values lead to similar output values.

Using these assumptions the output space is partitioned into different regions corresponding to different output linguistic values. How the space is partitioned is determined by the characteristic spacing parameters and the characteristic angle. The angle determines the slope of a line that goes through the origin on which seed points are placed. The positioning of the seed points is determined by a similar spacing method that is used to determine the center of the membership function.

Grid points are also placed in the output space and represent all the possible combination of input linguistic values. These are spaced in the same way as described previously. The rule-base is determined by calculating which seed-point is closest to each grid point. The output linguistic value representing the seed-point is set as the consequent of the antecedent represented by the grid point.

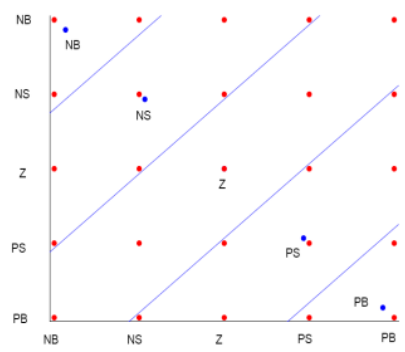

Fig.7. Seed points and grid points for rule-base construction

\begin{tabular}{|c|c|c|c|c|c|}
\hline 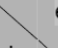 & NB & NS & Z & PS & PB \\
\hline NB & NB & NB & NS & NS & $\mathrm{Z}$ \\
\hline NS & NB & NS & NS & Z & PS \\
\hline $\bar{Z}$ & NS & NS & Z & PS & PS \\
\hline PS & NS & $\mathrm{Z}$ & PS & PS & PB \\
\hline PB & Z & PS & PS & PB & PB \\
\hline
\end{tabular}

Fig.8. Derived rule base

This is illustrated in Fig.7, which is a graph showing both the seed points (blue circles) and the grid-points (red circles). Fig.8 shows the derived rule base with the output as the control voltage variable. The lines on the graph delineate the different regions corresponding to the different consequents. The parameters for this example are 0.9 for both input spacing parameters, 1 for the output spacing parameter and a $45^{\circ}$ angle theta parameter.

\subsection{Parameter encoding}

To run a MPSO, suitable encoding needs to be carefully completed for each of the parameters and bounds. For this task the parameters given in Table 1 are used with the ranges and precision parameters that are shown. Binary encoding is used because it allows the MPSO more flexibility in searching the solution space thoroughly. The number of membership functions is limited to odd integers, which are inclusive between (3-9) 
when using the MPSO-based IPMC inverse fuzzy NARX11 model and between (3-5) when the MPSO-based IPMC inverse fuzzy NARX22 model identification is used. Experimentally, this was considered to be a reasonable constraint to apply. The advantage of doing this is that this parameter can be captured in just one to two bits per variable.

Two separate parameters are used for the spacing parameters. The first is within the range of $[0.1-1.0]$, which determines the magnitude and the second, which takes only the values -1

or 1 , is the power by which the magnitude is to be raised. This determines whether the membership functions compress in the center or at the extremes. Consequently, each spacing parameter can achieve a range of $[0.1-10]$. The precision required for the magnitude is 0.01 , which means that 8 bits are used in total for each spacing parameter. The scaling for the input variables is allowed to vary in the range of $[0-100]$, while that of the output variable is given a range of [0 1000].

Table 1. MPSO-based inverse fuzzy NARX model parameters used for encoding

\begin{tabular}{|l|c|c|c|}
\hline \multicolumn{1}{|c|}{ Parameter } & Range & Precision & No. of Bits \\
\hline Number of Membership Functions & $3-9$ & 2 & 2 \\
\hline Membership Function Scaling & $0.1-1.0$ & 0.01 & 7 \\
\hline Membership Function Spacing & $-1-1$ & 2 & 1 \\
\hline Rule-Base Scaling & $0.1-1.0$ & 0.01 & 7 \\
\hline Rule-Base Spacing & $-1-1$ & 2 & 1 \\
\hline Input Scaling & $0-100$ & 0.1 & 10 \\
\hline Output Scaling & $0-1000$ & 0.1 & 14 \\
\hline Rule-Base Angle & $0-2 \pi$ & $\pi / 512$ & 11 \\
\hline
\end{tabular}

\section{IDENTIFICATION RESULTS}

In general, the procedure which must be executed when attempting to identify a dynamical system consists of four basic steps.

STEP 1 (Getting Training Data)

STEP 2 (Select Model Structure )

STEP 3 (Estimate Model)

STEP 4 (Validate Model)

In Step 1, the identification procedure is based on the experimental input-output data values measured from the IPMC actuator system. The excitation input signal $\mathrm{u}(\mathrm{t})$ is chosen as a pseudo random binary sequence (PRBS). The PRBS signal proves to be the best efficient signal for identifying a highly nonlinear system. Figure 10 presents the PRBS inputs applied to the tested IPMC actuator system and the corresponding IPMC position output [mm]. 


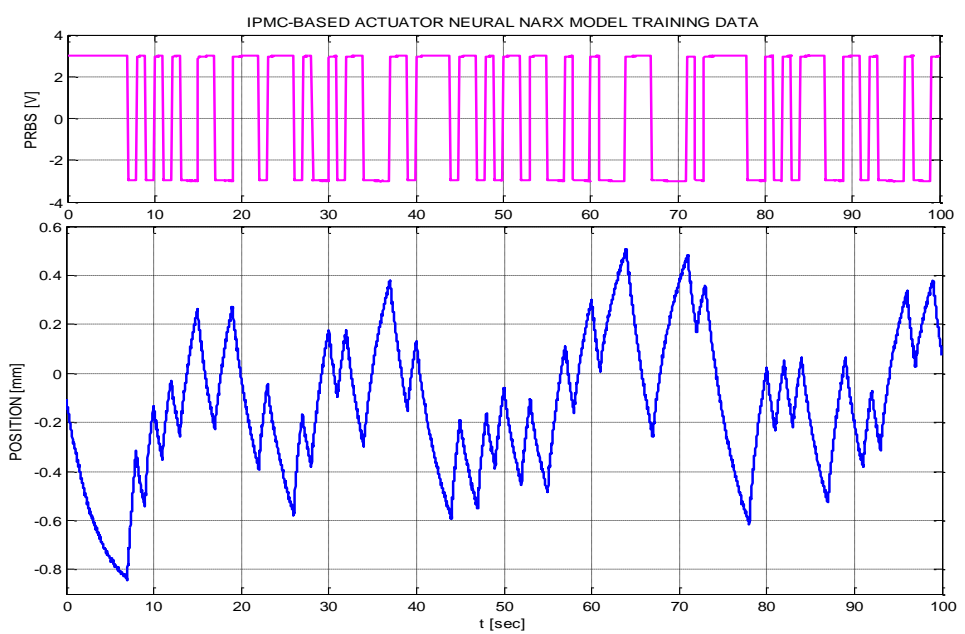

Fig.9. IPMC Actuator Inverse Fuzzy NARX Model Training data

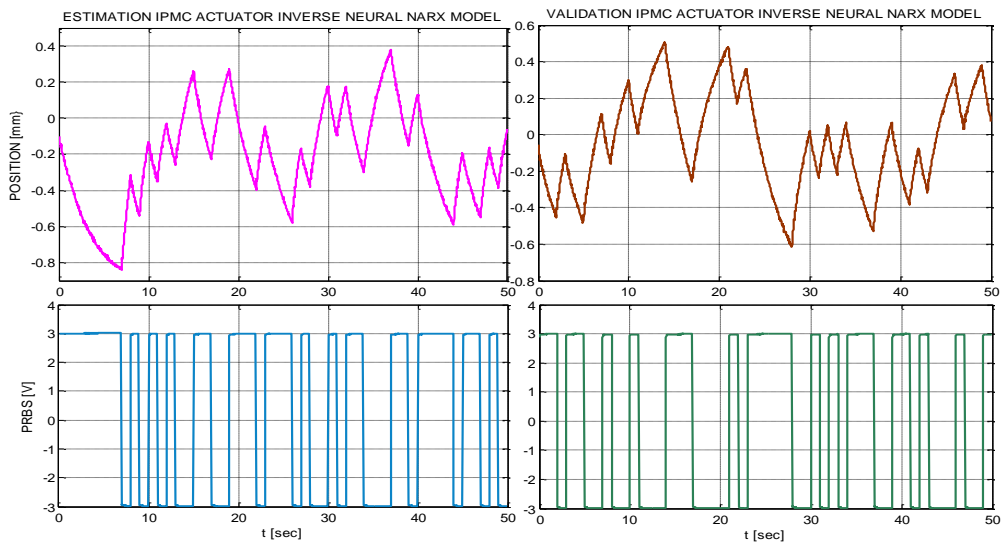

Fig. 10. Estimation and Validation Training data

This experimental PRBS input-output data is used for training and validating the Inverse fuzzy NARX model. The PRBS input and the IPMC actuator position output from (0-50) [s] being used for training, while PRBS input and the IPMC actuator position output from (50-100)[s] are used for validation purpose (see Figure 10).

The second step relates to select model structure. The proposed inverse fuzzy NARX11 (IFN11) and inverse fuzzy NARX22 (IFN22) model structures are attempted. Table 1 tabulates the IMNF model parameters that were used to encode the optimized input values of the PSObased identification and optimization algorithm. The block diagrams in Fig.1 and Fig.2 illustrate the identification scheme of two different IFN models.

The third step estimates values for the trained Inverse NARX11 model. The optimal fitness value to use for the MPSO-based optimization and identification process is calculated maximally based on Equation (7). 
The estimation result is presented in Fig.11 and $12($ with population $=20$ and generation $=100)$. These figures represent the fitness convergence values of the proposed IPMC IFN system which correspond to two different IFN models (Inverse fuzzy NARX11 and Inverse fuzzy NARX22 models) and all two were identified and optimized with MPSO identification method.

The fitness value of the proposed IPMC IFN model produces an excellent global optimal value (equal to 133200 with IFN11 and 164200 with
IFN22 model).

These good results are due to the proposed IFN model combines the extraordinary approximating capability of the fuzzy system with the powerful predictive and adaptive potentiality of the nonlinear NARX structure that is implied in the IFN model. Consequently, the MPSO-based IPMC IFN model addresses all of the nonlinear features of the IPMC actuator system that are implied in the input signals $\operatorname{PRBS}(\mathrm{z})[\mathrm{v}]$ and position $\mathrm{Y}(\mathrm{z}-1)$ $[\mathrm{mm}]$.

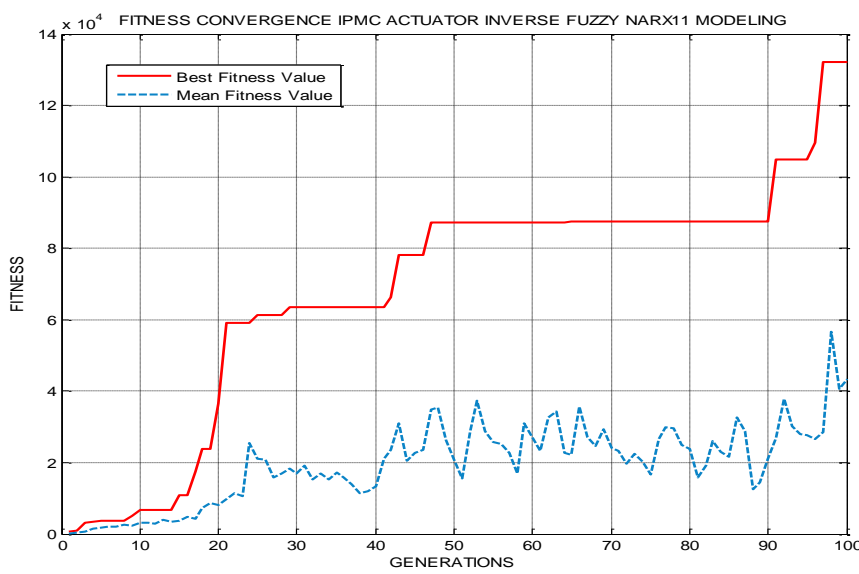

Fig.11. Fitness convergence of IPMC inverse fuzzy NARX11 model

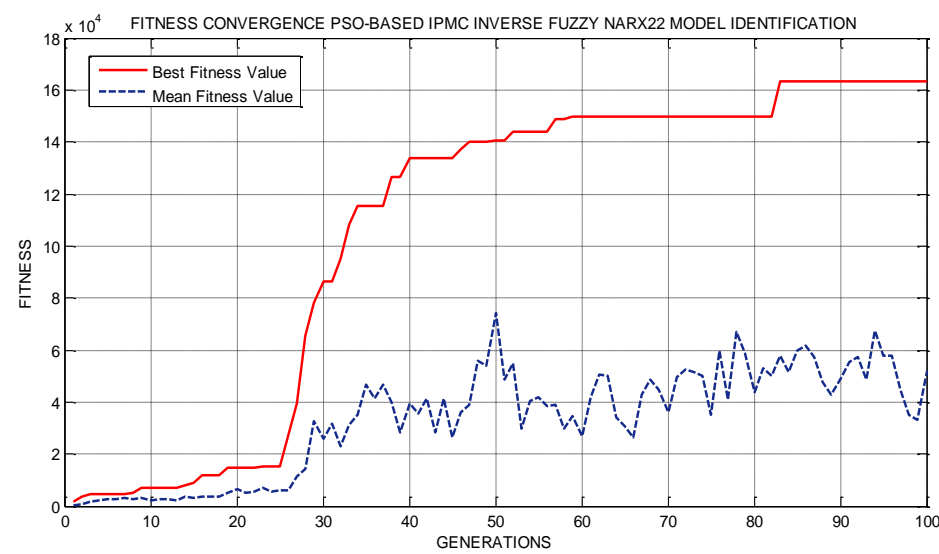

Fig.12. Fitness convergence of IPMC inverse fuzzy NARX22 model 
The last step relates to validate the resulting nonlinear IFN models. An excellent validating result demonstrates the performance of the resulting Inverse NARX Fuzzy model. The results of the MPSO-based IPMC actuator's NARX fuzzy model presented in Fig.14a and 14b obtain a very good range of error (error ranges are $<[ \pm 0.1[V]]$ for both of the IFN11 and IFN22 models).

The results show that with the same initial parameters for the MPSO-based identification method (including the population $=20$ and the generation=100), the proposed Inverse NARX Fuzzy model produces a very good fitness value (equal to 133200 with the inverse fuzzy NARX11 (IFN11) model and 164200 with IFN22 model). The compact structure of the Inverse fuzzy NARX11 model (with the number of membership functions (MF) of the two inputs and the one output equal to [7-9-5] is available to be applied in industrial practice. Consequently these results confirm the proposed Inverse NARX fuzzy model for use not only in modeling and identification but also in advanced control applications [20].

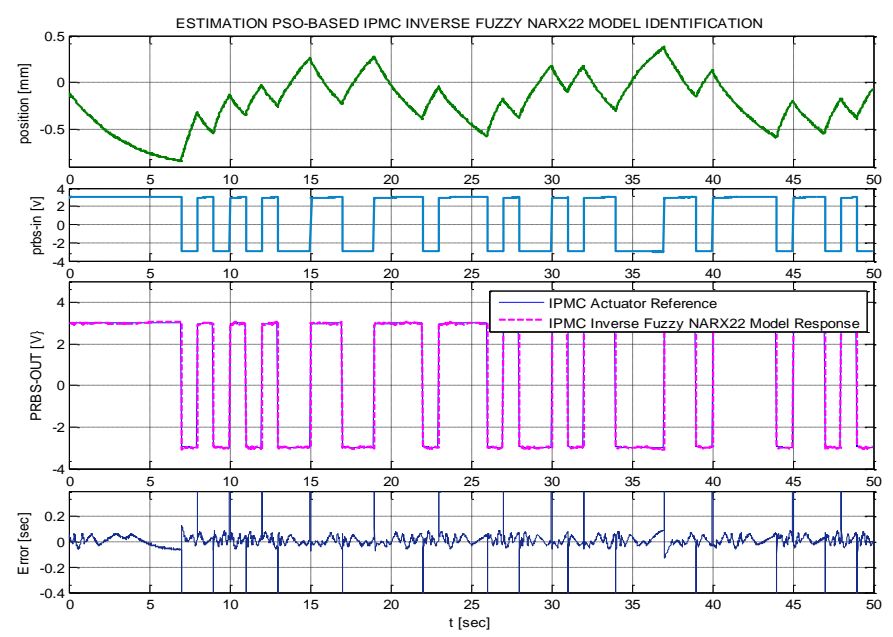

Fig.13. Estimation of proposed IPMC inverse fuzzy NARX22 Model

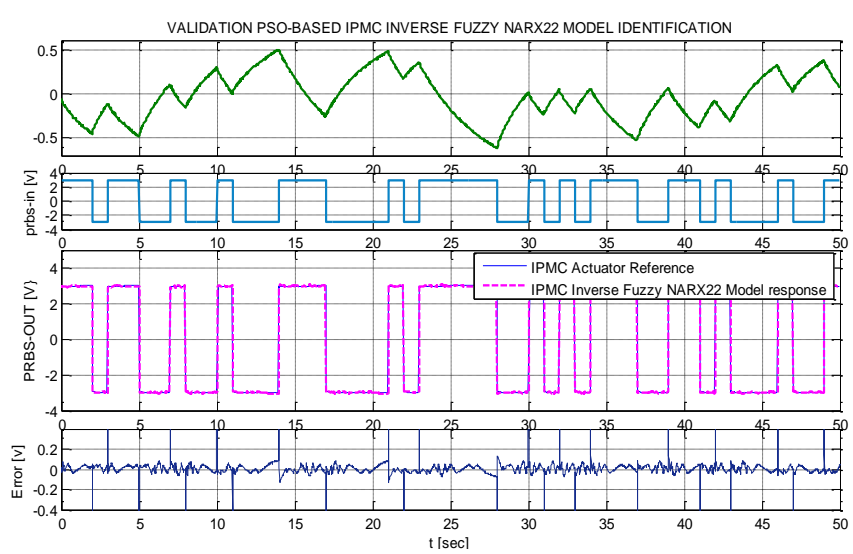

Fig.14a. Validation of IPMC inverse fuzzy NARX11 Model 


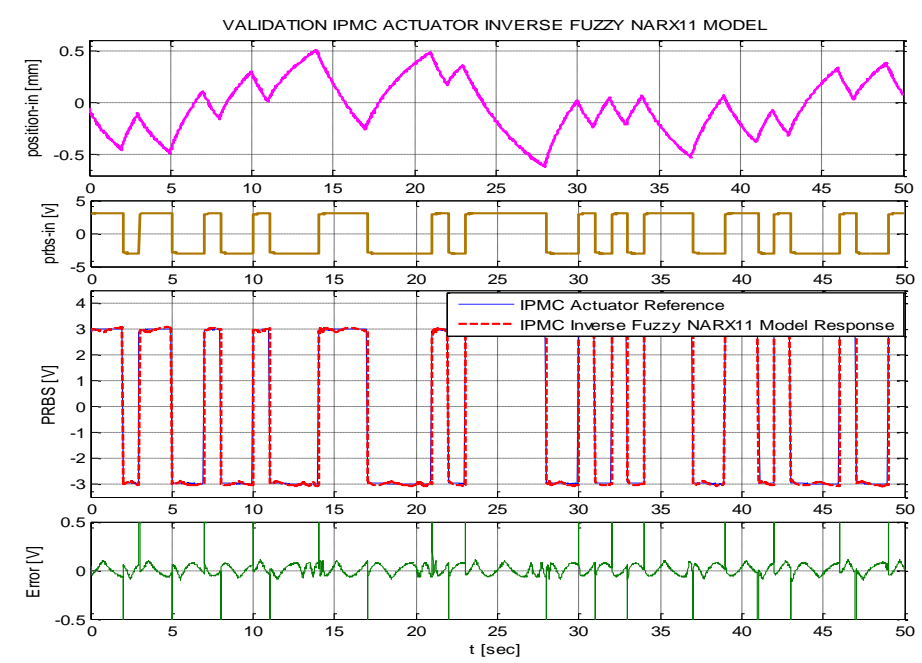

Fig.14b. Validation of IPMC inverse fuzzy NARX22 Model
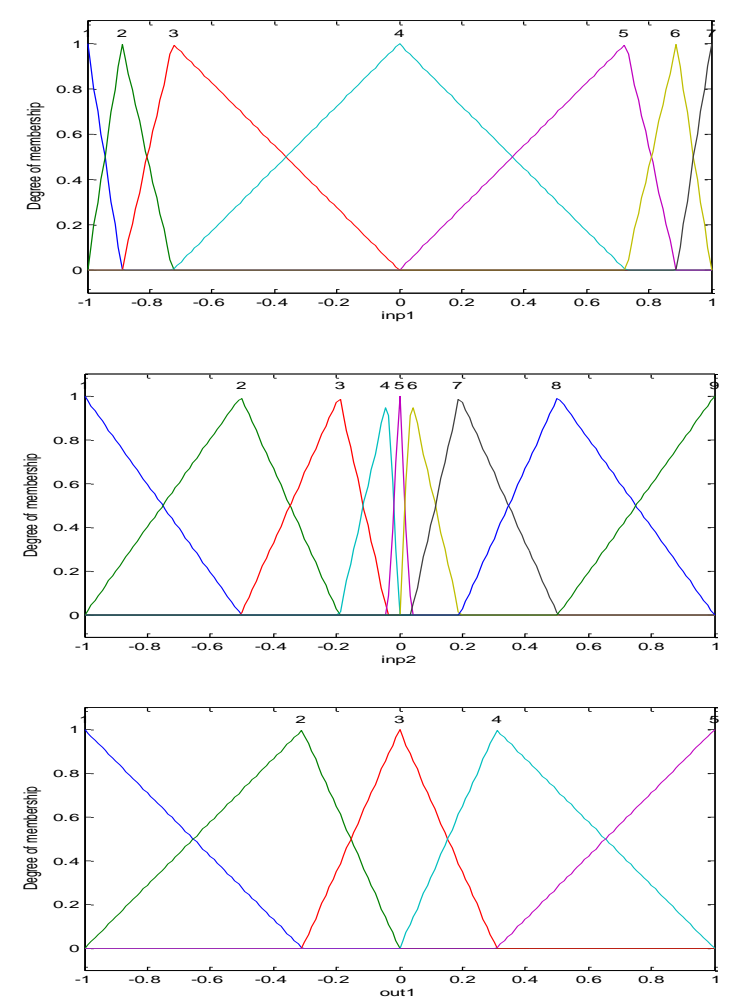

Fig.15. Inputs and Output IPMC fuzzy NARX11 MF

Figure 15 presents the two Inputs and the the surf-viewer of IPMC inverse fuzzy NARX11 Output of the IPMC fuzzy NARX11 membership and fuzzy NARX22 models' FIS structure. functions (MFs). Figures 16a and 16b introduce 


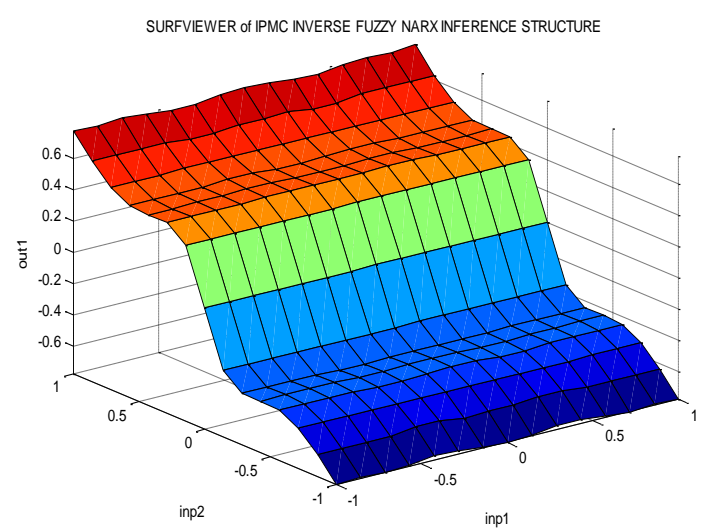

Fig.16a. Surf-viewer of IPMC inverse fuzzy NARX11 model FIS structure
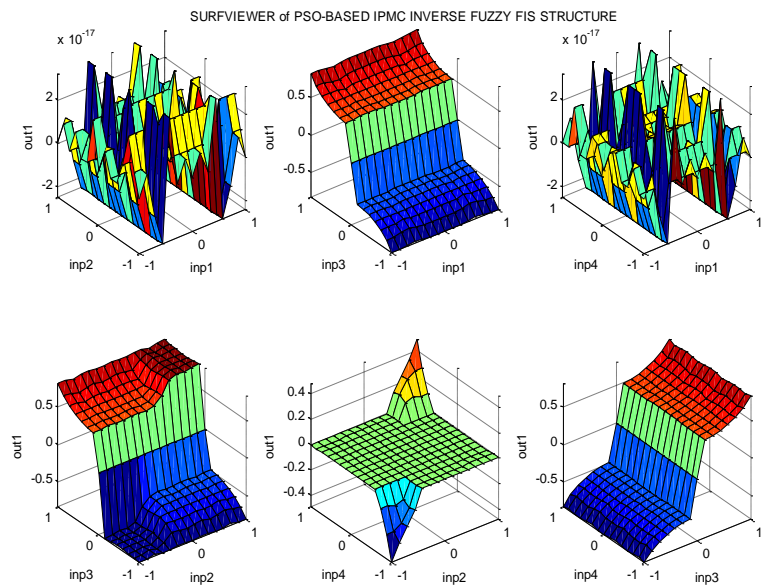

Fig.16b. Surf-viewer of IPMC inverse fuzzy NARX22 model FIS structure

Table 2. IPMC actuator inverse fuzzy NARX11 model rule-base

\begin{tabular}{|r|r|r|r|r|r|r|r|}
\hline & 1 & 2 & 3 & 4 & 5 & 6 & 7 \\
\hline 1 & 1 & 1 & 1 & 1 & 1 & 1 & 1 \\
\hline 2 & 2 & 2 & 2 & 2 & 2 & 2 & 2 \\
\hline 3 & 2 & 2 & 2 & 2 & 2 & 2 & 2 \\
\hline 4 & 3 & 3 & 3 & 3 & 3 & 3 & 3 \\
\hline 5 & 3 & 3 & 3 & 3 & 3 & 3 & 3 \\
\hline 6 & 3 & 3 & 3 & 3 & 3 & 3 & 3 \\
\hline 7 & 4 & 4 & 4 & 4 & 4 & 4 & 4 \\
\hline 8 & 4 & 4 & 4 & 4 & 4 & 4 & 4 \\
\hline 9 & 5 & 5 & 5 & 5 & 5 & 5 & 5 \\
\hline
\end{tabular}


Finally, Table 2 tabulated the resulting identified fuzzy rule-bases of the IPMC actuator inverse fuzzy NARX11 model.

\section{CONCLUSIONS}

In this paper a new approach of inverse dynamic fuzzy NARX model firstly utilized in modeling and identification of the IPMC actuator. Training and testing results show that the newly proposed inverse dynamic fuzzy NARX model optimized by the novel MPSO algorithm presented in this study can be used in online control with better dynamic property and strong robustness. This resulting proposed intelligent model is quite suitable to be applied for the modeling, identification and control of various complex plants, including linear and nonlinear process without regard greatly change of external environments.

ACKNOWLEDGMENTS: This research is funded by DCSELAB and by Vietnam National Foundation for Science and Technology Development (NAFOSTED) under grant number 107.04-2012.23.

\section{Nhận dạng mô hình động học của bộ truyền động IPMC dùng mô hình mờ fuzzy NARX được tối ưu bằng $\mathrm{PSO}$}

- Hồ Phạm Huy Anh

FEEE, Trường Đại học Bách khoa, ĐHQG-HCM

- Nguyễn Thanh Nam

DCSELAB, Trường Đại học Bách khoa, ĐHQG-HCM

\section{TÓM TÁ́T:}

Bài báo giới thiệu mô hình mờ fuzzy NARX lần đầu được dùng để nhận dạng động học ngược bộ truyền động IPMC. Các biến động do lực tiếp xúc cũng nhu các hiệu ứng chéo phi tuyến của IPMC sẽ được nhận dạng đầy đủ bởi mô hình mờ fuzzy NARX thong qua dũ̃ liệu huấn luyện lấy từ thực nghiệm. Bài báo cũng trình bày cách khai thác thuật toán bầy đàn nâng cao (modified particle swarm optimization MPSO) để tối ưu thông số của mô hình mờ fuzzy NARX dùng nhận dạng hệ truyền động IPMC phi tuyến. Kết quả cho thấy mô hình mờ fuzzy NARX model được tối ưu bởi thuật toán bầy đàn nâng cao (MPSO) cho tính năng và độ chính xác vượt trội so với các mô hình nhận dạng đã có.

Từ Khóa: bộ truyền động IPMC, thuật toán tối ưu bầy đàn nâng cao (modified particle swarm optimization - MPSO), mô hình mờ fuzzy NARX, nhận dạng động học ngược. 


\section{REFERENCES}

[1]. M. Sadeghipour, R. Salomon, and S. Neogi, "Development of a novel electrochemically active membrane and 'smart' material based vibration sensor/damper," Smart Materials and Structures, vol. 1, no. 1, pp. 172-179, May 1992.

[2]. M. Shahinpoor, "Conceptual design, kinematics and dynamics of swimming robotic structures using ionic polymer gel muscles," Smart Materials and Structures, vol. 1, no. 1, pp. 91-94, May 1992.

[3]. K. Oguru, Y. Kawami, and H. Takenaka, "Bending of an ion-conducing polymer film-electrode composite by an electric stimulus at low voltage," Trans. Journal of Micromachine Society, vol. 5, pp. 27-30, September 1992.

[4]. S. Tadokoro, S. Yamagami, T. Takamori, and K. Oguro, "Modeling of Nafion-Pt composite actuators (ICPF) by ionic motion," in Proceedings of SPIE, Smart Structures and Materials, vol. 3987, pp. 92-102, March 2000.

[5]. Y. Bar-Cohen, S. Sherrit, and S. Lih, "Characterization of the electromechanical properties of EAP materials," in Proceedings of EAPAD, SPIE's 8th AnnualInternational Symposium on Smart Structures and Materials, pp. 4326-4343, March 2001.

[6]. R. Kanno, S. Tadokoro, M. Hattori, T. Takamori, M. Costaftis, and K. Oguro, "Dynamic model of ICPF (Ionic Conducting Polymer Film) actuator," in Proceedings of IEEE International Conference on Systems, Man, and Cybernetics: Intelligent Systems for the 21st Century, vol. 1, pp. 177-182, October 1995.
[7]. R. Kanno, S. Tadokoro, M. Hattori, T. Takamori, M. Costaftis, and K. Oguro, "Modeling of ICPF (Ionic Conducting Polymer Film) actuator," in Proceedings of the 1995 IEEE 21st International Conference on Industrial Electronics, Control, and Instrumentation, vol. 2, pp. 913-918, November 1995.

[8]. R. Kanno, S. Tadokoro, and T. Takamori, "Linear approximate dynamic model of ICPF," in Proceedings of the IEEE International Conference on Robotics and Automation, vol. 1, pp. 219-225, April 1996.

[9]. N. Bhat and W. -J. Kim, "Precision force and position control of ionic polymer metal composite," Journal of Systems and Control Engineering, vol. 218, no. 6, pp. 421-432, June 2004.

[10]. N. Bhat, Modeling and Precision Control of Ionic Polymer Metal Composite, Master's Thesis, Texas A\&M University, August 2003.

[11]. N. Bhat and W. -J. Kim, "System identification and control of ionic polymer metal composite," in Proceedings of SPIE, Smart Structures and Materials, vol. 5049, pp. 526-535, March 2003.

[12]. K. Newbury and D. J. Leo, "Modeling of electromechanical transduction in ionic polymer materials," in Proceedings of ASME International Mechanical Engineering Congress \& Exposition, pp. 47-57, November 2002.

[13]. Zhou C L, Low K H (2012) Design and Locomotion Control of a Biomimetic Underwater Vehicle with Fin Propulsion. IEEE/ASME T. Mech. 17(1): 25-35.

[14]. Chen Z, Shatara S, Tan X B (2010) Modeling of Biomimetic Robotic Fish 
Propelled by An Ionic Polymer-Metal Composite Caudal Fin. IEEE/ASME T. Mech. 15(3): 448 - 459.

[15]. Maxwell J Fleming et al, 2013, "Mitigating IPMC back relaxation through feedforward and feedback control of patterned electrodes", Smart Mater. Struct. Vol.21, n.8, doi:10.1088/09641726/21/8/085002.

[16]. Wang T, Shen Q, Wen L, et al. (2012) On the Thrust Performance of an Ionic Polymer-metal Composite Actuated Robotic Fish: Modeling and Experimental Investigation. Sci. China Tech. Sci. 55: 3359-69

[17]. Shen Q, Wang T, Wen L, et al. (2013) On the Thrust Efficiency of an IPMC-actuated Robotic Swimmer: Dynamic Modeling and Experimental Investigation. Proc. Int. O shore and Polar Engineering Conf. pp.55662.

[18]. Roy Dong and Xiaobo Tan, (2013), "Modeling and open-loop control of IPMC actuators under changing ambient temperature", Smart Mater. Struct. Vol.21, n.6, doi:10.1088/0964-1726/21/6/065014.

[19]. M. Farid,, Zhao Gang, Tran Linh Khuong, Z. Z. Sun, "Forward Kinematic Modeling and Simulation of Ionic Polymer Metal Composites (IPMC) Actuators for Bionic Knee Joint", Advanced Materials Research, Feb. 2014, Vol. 889 - 890, pp. 938-941.

[20]. K.K. Ahn, and H.P.H. Anh, "A New Approach of Modeling Identification and Hybrid Feed-Forward-PID Control of The Pneumatic Artificial Muscle (PAM) Robot Arm using Inverse NARX Fuzzy Model and Genetic Algorithm," Journal of Engineering Applications of Artificial Intelligence, EAAI, Elsevier, Volume 24, Issue 4, June 2011, Pages 697-716.
[21]. K.K Ahn, H.P.H. Anh, "A new approach of modeling and identification of the pneumatic artificial muscle manipulator based on recurrent neural network," In Proceedings IMechE, Part I: Journal of Systems and Control Engineering, 2007, 221(I8), 1101-1122.

[22]. Doan Ngoc Chi Nam, Kyoung Kwan Ahn, "Identification of an ionic polymer metal composite actuator employing Preisach type fuzzy NARX model and Particle Swarm Optimization", Sensors and Actuators A: Physical, Vol. 183, Aug. 2012, pp. 105-114

[23]. Nam Doan Ngoc Chi, Truong Dinh Quang, Jong Il Yoon, Kyoung Kwan Ahn, "Identification of ionic polymer metal composite actuator employing fuzzy NARX model and Particle Swam Optimization", International Conference on Control, Automation and Systems (ICCAS), 2011 11th , pp. 1857 - 1861.

[24]. L. Yao, and P. Huang, "Learning of Hybrid Fuzzy Controller for the Optical Data Storage Device," IEEE/ASME Transactions on Mechatronics, Vol. 13, no. 1, Feb. 2008, pp.3-13.

[25]. S. Su and F. P. Yang, "On the Dynamical Modeling With Neural Fuzzy Networks," IEEE Transactions on Neural Networks, Vol. 13, no. 6, Nov. 2002, pp.1548-1553.

[26]. Y. S. Hong, M. R. Rosen and R. R. Reeves, "Dynamic Fuzzy Modeling of Storm Water Infiltration in Urban Fractured Aquifers," JOURNAL OF HYDROLOGIC ENGINEERING, 2002, pp. 380-391.

[27]. K.K. Ahn, and H.P.H. Anh, "Inverse Double NARX Fuzzy Model for System Identification," IEEE/ASME Journal of MECHATRONICS, Vol. 15, Issue 1, pp. 136-148. 
[28]. [28] R.J. Wai, K.H. Su, "Supervisory control for linear piezoelectric ceramic motor drive using genetic algorithm," IEEE Trans. on Industrial Electronics, vol. 53, no. 2, pp. 657-673, Apr 2006.

[29]. F.J. Lin, P.K. Huang, W.D. Chou, "Recurrent-Fuzzy-Neural-NetworkControlled Linear Induction Motor Servo Drive Using Genetic Algorithms," IEEE Trans. on Industrial Electronics, vol. 54, no. 3, pp. 1449-1461, June 2007.

[30]. S.K. Oh, W. Pedrycz, H.S. Park, "A New Approach to the Development of Genetically Optimized Multilayer Fuzzy Polynomial Neural Networks," IEEE Trans. on Industrial Electronics, vol. 53, no. 4, pp. 1309- 1321, Aug 2006.

[31]. S.-B. Roh, W. Pedrycz, S.K. Oh, "Genetic Optimization of Fuzzy Polynomial Neural
Networks," IEEE Trans. on Industrial Electronics, vol. 54, no. 4, pp. 2209-2218 , Aug 2007.

[32]. J. Kennedy, R. Eberhart, "Particle Swarm Optimization", Proc. IEEE Int. Conf. on Neural Network, Vol. 4, 1995, pp. 1942 1948.

[33]. H. Yoshida, K. Kawata, Y. Fukuyama, S. Takayama, Y. Nakanishi, “A Particle Swarm Optimization for Reactive Power and Voltage Control Considering Voltage Security Assessment", IEEE Trans. on Power Systems, Vol. 15, No. 4, Nov. 2000, pp. $1232-1239$.

[34]. Z.L. Gaing, "A Particle Swarm Optimization Approach for Optimum Design of PID Controller in AVR System", IEEE Trans. Energy Conversion, Vol. 19, No. 2, June 2004, pp. $384-391$ 